\title{
COLLISIONAL EFFECTS ON THE DENSITY PROFILES OF SPHERICAL GALAXIES
}

\author{
L.A. Aguilar \\ Harvard-Smithsonian Center for Astrophysics \\ 60 Garden St., Cambridge MA 02138 USA \\ S.D.M. White \\ Steward Observatory \\ University of Arizona, Tucson AZ 85721 USA
}

ABSTRACT. We use N-body simulations to study the time evolution and the final shape of the density profiles of non-rotating spherical galaxies that have undergone a tidal encounter. We consider models with de Vaucouleurs and King surface density profiles and with isotropic, tangential and radially biased velocity distributions.

King models lose their tidal radius and develop an extended tail very similar to an $r^{1 / 4}$-law. De Vaucouleurs models, on the other hand, are very robust and are still good fits to the remnants of even very strong encounters resulting in a mass loss of $50 \%$. Although an extended tail of the profile is always regenerated after an encounter, the isophotal radius of the galaxy almost always decreases.

The tidal classes defined by Kormendy (1977) can be interpreted as resulting from a transient radial mixing of stars inside a galaxy after an encounter, and can be used to estimate the time since last encounter.

A galaxy that has suffered tidal encounters does not show any "collision signature" in its luminosity profile or shape of isophotes; the effects of tidal encounters can only be recognized immediately after a close passage, or by a statistical study of galaxy properties of a sample of galaxies.

NUMERICAL EXPERIMENTS. Our initial conditions are 3000-particle random realizations of spherical models with de Vaucouleurs and King surface density profiles. In order to test possible effects due to internal kinematics, three different velocity distributions have been used for the de Vaucouleurs models: isotropic, radially biased, and purely circular orbits. Only isotropic velocity distributions have been used for the King models. All models have the same binding energy.

We have used an N-body code that approximates the forces between the particles in a self-gravitating target galaxy as an expansion in spherical harmonics up to quadrupolar terms. The perturbing galaxy is modelled as a rigid potential that corresponds to a galaxy identical to the target galaxy but with a different mass. For more details see Aguilar and White (1985).

RESULTS. We have calculated the surface density profile projected on the orbital plane of the encounter for all our experiments. Only bound particles have been considered. Figure 1 presents the evolution of the surface density profile for a strong encounter that results in $\mathbf{4 0 \%}$ mass loss to the target galaxy. 

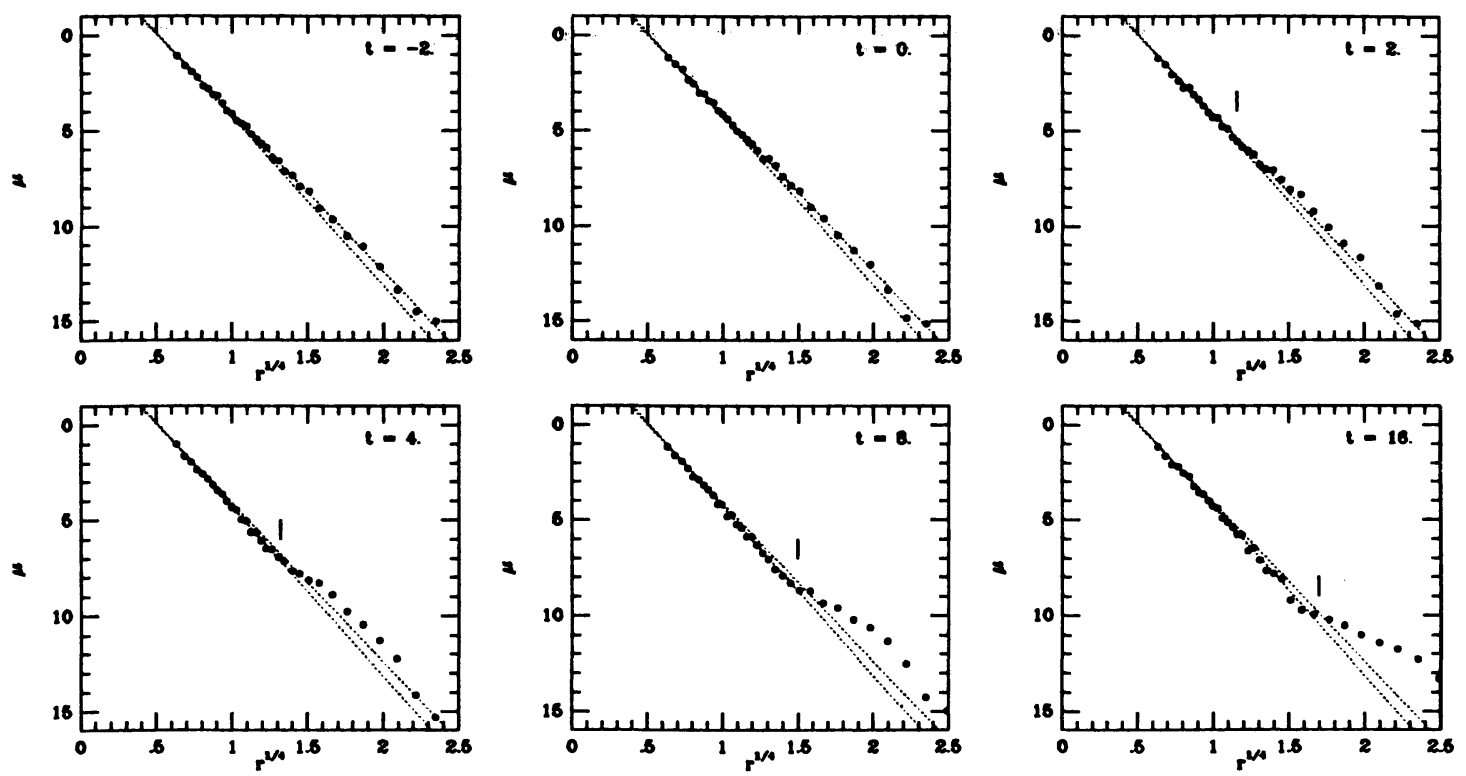

Figure 1. Evolution of the surface density profile of an $r^{1 / 4}$ model after a strong encounter $\left(M_{p} / M_{\text {target }}=1.9, p / r_{e}=1.0\right.$, and $\left.v_{\text {coll }} / v_{r m s}=2.2\right)$. Time is given for each frame in crossing times ( $t \equiv 0$ at the instant of closest approach). Upper and lower dotted lines in all frames are $r^{1 / 4}$-laws fitted to the initial and final $(t=80)$ frames respectively. The solid tick mark in several frames lies at the radius where the local $t_{\text {cross }}$ equals the time elapsed since closest approach.

The evolution of the density profile is quite simple. As time passes, the innermost regions move to the line defined by the fit to the final conditions, while the outer regions produce a "luminous" excess above both, initial and final profiles. The boundary between these two regions evolves on the local dynamical timescale, proving that the bump in the profile consists of particles being radially mixed onto less bound orbits. Notice that the profile at $t=16 t_{\text {cross }}\left(\sim 2.6 \times 10^{8}\right.$ years for a galaxy with $r_{e}=2 \mathrm{kpc}$ and $v_{r m s}=250 \mathrm{~km} / \mathrm{s}$ ) is indistinguishable, within the numerical resolution of the simulation, from an $r^{1 / 4}$-law for $r<5 r_{e}$. The "tidally distended" objects found by Kormendy have very similar profiles, and if produced by an encounter, indicate a very recent event; we note that Kormendy's T3 tidal class is defined by the presence of a nearby luminous companion.

Collisions using other initial conditions show a similar evolution on the same timescale, the amplitude of the bump being a function of encounter parameters. King models develop an extended tail in the profile and resemble an $r^{1 / 4}$ - law. We conclude that galaxies that have suffered tidal encounters do not show any encounter signature within its observable region, unless observed immediately after the encounter. A statistical study of the relationship between profile shape and nearest neighbor distance would be very useful to see whether "tidal distension" is consistent with the short timescales we infer from our models.

\section{REFERENCES}

Aguilar, L.A. and White, S.D.M., 1985. Astrophys. J., 295, 374.

Kormendy, J. 1977. Astrophys. J., 218, 333. 